\title{
ROBUST FRACTIONAL ADAPTIVE CONTROL BASED ON THE STRICTLY POSITIVE REALNESS CONDITION
}

\author{
SAMIR LADACI * , ABDELfatah CHAREF ${ }^{* *}$, JEAN JACQUES LOISEAU *** \\ * Department of Electrical Engineering \\ University of the 20th August 1955 of Skikda, BP 26, Skikda 21000, Algeria \\ e-mail: samir_ladaci@yahoo.fr \\ ** Department of Electronics \\ University of Mentouri of Constantine, Route de Ain El-bey, Constantine 25000, Algeria \\ e-mail: afcharef@yahoo.com \\ *** IRCCyN-Ecole Centrale de Nantes \\ 1, rue de la Noë, BP 92101 Nantes, 44321, France \\ e-mail: Jean-Jacques . Loiseau@irccyn.ec-nantes. fr
}

\begin{abstract}
This paper presents a new approach to robust adaptive control, using fractional order systems as parallel feedforward in the adaptation loop. The problem is that adaptive control systems may diverge when confronted with finite sensor and actuator dynamics, or with parasitic disturbances. One of the classical robust adaptive control solutions to these problems makes use of parallel feedforward and simplified adaptive controllers based on the concept of positive realness. The proposed control scheme is based on the Almost Strictly Positive Realness (ASPR) property of the plant. We show that this condition implies also robust stability in the case of fractional order controllers. An application to Model Reference Adaptive Control (MRAC) with a fractional order adaptation rule is provided with an implementable algorithm. A simulation example of a SISO robust adaptive control system illustrates the advantages of the proposed method in the presence of disturbances and noise.
\end{abstract}

Keywords: positive realness, robust adaptive control, fractional adaptive control, model reference adaptive control, feedforward, fractional calculus.

\section{Introduction}

Adaptive control has proven to be a good control solution for partially unknown systems or systems with slowly varying parameters. In this domain, Model Reference Adaptive Control (MRAC) has become very popular since it presents a very simple algorithm with easy implementation and does not require identifiers or observers in the control loop (Åström and Wittenmark, 1995; Landau, 1979). However, such an algorithm shows its limits in noisy or disturbed environments, which may make it inefficient or uncompetitive. Unfortunately, very few industrial control processes are not subject to these practical problems, which can damage the quality of product and good process operation.

The use of simple parallel feedforward in the adaptation loop has appeared as a robust solution since the 1980s. Many works have used this approach towards robust control systems (Bar-Kana, 1987; Naceri and Abida, 2003)

In the last decade great attention has been paid to fractional order systems, which have shown good robustness performances. Several robust control methods based on these systems were developed, e.g., CRONE control (Oustaloup et al., 1998; Sabatier et al., 2002) and fractional adaptive control (Vinagre et al., 2002; Ladaci and Charef, 2006; Ladaci et al., 2008).

In this paper we present a fractional robust adaptive control solution for disturbed applications, based on the idea of Bar-Kana (Bar-Kana, 1987), which uses the basic stabilizability property of the plant and simple parallel feedforward in order to satisfy the desired "almost positive realness" condition that can guarantee robust stability of the nonlinear adaptive controller. 
The main contribution of this work is the improvement of feedforward approach robust performances by using fractional order filters. In the proposed adaptive control scheme, the process transfer function is augmented by an additional, appropriately designed, fractional order transfer function connected in parallel. The control of the augmented process is achieved in the fractional order MRAC structure (Ladaci and Charef, 2006). This result is illustrated by a simulation example of a test in bad realistic conditions such as the finite bandwidth of actuators, input and output disturbances and no assumed natural damping.

This paper is structured as follows: In Section 2, definitions of fractional order systems are presented. Section 3 introduces the principles of robust adaptive control based on the concept of a 'positive realness' condition, and then the main result in the fractional order case is presented in Section 4. The implementation in a model reference adaptive control scheme is introduced in Section 5, and a simulation example is given in Section 6. The paper is concluded in Section 7.

\section{Fractional order systems}

The Bode plot analysis of many natural processes, e.g., transmission lines, dielectric polarisation impedance, interfaces, cardiac rhythm, spectral density of physical wave, some types of noise (Brin, 1962; Podlubny, 1999a; Miller and Ross, 1993), often reveals a fractional slope. This type of process is known as a fractional order system. During the last decade, close attention has been paid by researchers to the study of these systems (Sun and Charef, 1990) and their application in control systems (Oustaloup et al., 1998; Charef, 2006; Ladaci and Charef, 2006; Ladaci et al., 2008; Ladaci and Moulay, 2008). A SISO fractional order system can be represented by the following transfer function:

$$
X(s)=\frac{b_{m} s^{\beta_{m}}+b_{m-1} s^{\beta_{m-1}}+\cdots+b_{0} s^{\beta_{0}}}{a_{n} s^{\alpha_{n}}+a_{n-1} s^{\alpha_{n-1}}+\cdots+a_{0} s^{\alpha_{0}}},
$$

where $\alpha_{i}$ and $\beta_{j}$ are real numbers such that

$$
\left\{\begin{array}{l}
0 \leq \alpha_{0}<\alpha_{1}<\cdots<\alpha_{n}, \\
0 \leq \beta_{0}<\beta_{1}<\cdots<\beta_{m},
\end{array}\right.
$$

and $s$ is the Laplace operator.

For the purpose of this work, let us introduce the following definitions:

Definition 1. The fractional order transfer function $X(s)$ given in (1) is called proper if $\beta_{m} \leq \alpha_{n}$. It is called stricly proper if $\beta_{m}<\alpha_{n}$.

Definition 2. (Desoer and Vidyasagar, 1975) The fractional order transfer function Matrix $M_{X}(s)$ whose elements are of the form (1) is proper (respectively strictly proper $)$ if and only if all elements of $M_{X}(s)$ are bounded at $\infty$ (resp. tend to zero at $\infty$ ).

In the sequel we use the description of a single pole fractional order process in frequency domain given by the following equation:

$$
Y(s)=\frac{1}{\left(s+p_{T}\right)^{\alpha}},
$$

where $\alpha$ is the fractional exponent $(0 \leq \alpha \leq 1)$ and $p_{T}$ is the fractional pole which is the cut frequency.

Many previous works indicated that fractional systems present the best qualities regarding response time and transition dynamic stability (Sun and Charef, 1990). The whole control theory developed by Oustaloup, especially on CRONE control, was based on the robustness of fractional order systems in the presence of uncertainties and perturbations (Oustaloup, 1991).

\section{Concept of the positive realness condition}

Robustness is defined relative to a certain property and a set of models. A property (generally, stability or the performance level) is said to be robust if all the models belonging to the set satisfy it. Robust adaptive stabilization means that all values involved in the adaptation process, namely, states, gains and errors, are bounded in the presence of any bounded input commands and input or output disturbances (Bar-Kana and Kaufman, 1985; Kwan et al., 2001).

In this paper we are interested in a particular configuration of feedforward controllers combined with MRAC control and fractional order systems yielding a fractional robust adaptive control method. The use of simple feedforward in the adaptation loop (see Fig. 4) improves the robust stability of the control system. This approach is based on the concept of the "positive realness" condition, which plays an important role in the stability analysis of a large class of nonlinear systems, including also adaptive systems.

The definition of PR and SPR transfer functions is derived from network theory. That is, a PR (resp. SPR) rational transfer function can be realized as the driving point impedance of a passive (resp. dissipative) network. Conversely, a passive (resp. dissipative) network has a driving point impedance that is rational and PR (resp. SPR). A passive network is the one that does not generate energy, i.e., a network consisting only of resistors, capacitors and inductors. A dissipative network dissipates energy, which implies that it is made up of resistors as well as capacitors and inductors that are connected in parallel with resistors (Ioannou and Sun, 1996).

Definition 3. A system $S$ with a transfer function matrix $G_{s}(s)$ is passive and stable if and only if $G(s)$ is positivereal (PR) (Anderson and Vongpanitlerd, 1973). 
Definition 4. An $m \times m$ transfer function matrix $G_{s}(s)$ is called strictly positive real (SPR) if (Landau, 1979):

1. All elements of $G_{s}(s)$ are analytic in $\Re(s) \geq 0$.

2. $G_{s}(s)$ is real for real $s$.

3. $G_{s}(s)+G_{s}^{T *}(s)>0$ for $\Re(s) \geq 0$ and finite $s$.

An equivalent definition is as follows:

Definition 5. A transfer function matrix $G_{s}(s)$ is strictly positive real (SPR) if and only if (Anderson and Vongpanitlerd, 1973):

1. $G_{s}(s)$ is a strictly stable transfer function matrix.

2. $\Re\left[G_{s}(s)\right]>0$ along the $j \omega$ axis.

We also show that for a fractional order transfer function matrix $G_{s}(s)$,

$$
G_{s}(s) \text { is } \mathrm{SPR} \Leftrightarrow G_{s}^{-1}(s) \text { is } \mathrm{SPR} .
$$

This is a generalization of the result obtained in (Shaked, 1977) for an integer order transfer function matrix.

Indeed, by using the SPR property, if we write (BarKana, 1989) (using $A, B$ instead of $A(s), B(s)$ for simplificity),

$$
G_{s}(s)=A+j B \Rightarrow G_{s}^{T *}(s)=A^{T}-j B^{T} .
$$

Since, by Definition 4,

$$
G_{s}(s)+G_{s}^{T *}(s)=A+A^{T}+j\left(B-B^{T}\right)>0,
$$

we get $B=B^{T}$ and $A>0$ (not necessarily symmetric). Then, whenever

$$
\Re\left[G_{s}(s)\right]=A>0,
$$

we get

$$
\begin{aligned}
G_{s}^{-1}(s)= & \left(A+B A^{-1} B^{T}\right)^{-1} \\
& -j A^{-1} B\left(A+B A^{-1} B^{T}\right)^{-1}
\end{aligned}
$$

and

$$
\Re\left[G_{s}^{-1}(s)\right]=\left(A+B A^{-1} B^{T}\right)^{-1}>0 .
$$

Now, recall the following proposition (Shaked, 1977):

Proposition 1. The finite zeros of any passive system $S$ lie in the closed left half of the complex plane.

From this and Definition 3 , since $G_{s}(s)$ is SPR, we deduce that $G_{s}^{-1}$ is strictly stable. This fact combined with (4) and Definition 5 proves (3).
Definition 6. (Bar-Kana, 1987) Let $G_{a}(s)$ be an $m \times m$ transfer matrix. Assume that there exists a positive definite constant gain matrix, $\tilde{K}_{e}$, such that the closed-loop transfer function

$$
G_{c}(s)=\left[I+G_{a}(s) \tilde{K}_{e}\right]^{-1} G_{a}(s)
$$

is SPR. $G_{a}(s)$ is called almost strictly positive real (ASPR).

All the above algebraic manipulations, as was done to obtain (3) and Definitions 4 and 6, apply to fractional systems as well. Below we generalize the result of (BarKana, 1989) to the fractional order case.

Lemma 1. Let a fractional order transfer function matrix $G_{a}(s)$ be ASPR and let $\tilde{K}_{e}$ be any gain that satisfies (5). Then $G_{c}(s)$ is SPR for any gain $K_{e}$ that satisfies $K_{e}>$ $\tilde{K}_{e}$

It is obvious that ASPR fractional order systems, which are minimum phase proper systems, maintain stability with high gains. The high gain stability is important when nonstationary or nonlinear (adaptive) control is used, because the robustness of the control system is maintained if, due to specific operational conditions, the time-varying gains become too large.

Now, if we consider a fractional order proper or strictly proper SPR transfer matrix $G_{s}(s)$, then the following statements are equivalent:

$$
\begin{array}{rlrl}
G_{s}(s) & =\left[I+G_{a}(s) K_{e}\right]^{-1} G_{a}(s) & & \text { is SPR, } \\
G_{s}(s) & =\left[I+G_{a}(s) K_{e}\right]^{-1} & & \text { is SPR, } \\
G_{s}^{-1}(s) & =G_{a}^{-1}(s)+K_{e} & \text { is SPR, } \\
\Re\left[G_{a}^{-1}(s)\right. & \left.+K_{e}\right]_{\Re(s) \geq 0}>0 & \\
G_{s}^{-1}(s) & \text { is asymptotically stable and } \\
K_{e} & \text { is sufficiently large. }
\end{array}
$$

Since there exists $M$ such that $\Re\left[G_{a}^{-1}(s)\right]_{\Re(s) \geq 0}>M>$ $-\infty$, any $K_{e}>-M$ will yield (Bar-Kana, 1989)

$$
\begin{array}{cl}
G_{a}(s) & \text { is strictly minimum phase and } \\
K_{e} & \text { is sufficiently large. }
\end{array}
$$

\section{Remarks.}

1. Any ASPR plant must also be proper.

2. The open loop is not necessarily stable (the plant will actually be stabilized by the fictitious gain $K_{e}$ ). However, all zeros must be placed in the left half plane. The plant must be minimum phase to obtain positivity.

3. We can easily show (Bar-Kana, 1987) that if a system is ASPR, then it can be stabilized by any constant or time variable output gain $K_{e}$, if it is large enough, i.e., $K_{e}>\tilde{K}_{e}$. 
But in this method, instead of using high gain regulation, we will use a simple parallel feedforward configuration which can by analogy satisfy the positive realness conditions.

The idea of using feedforward in parallel with the controlled plant is based on the following result:

Lemma 2. (Bar-Kana, 1989) Let the plant be described by an $m \times m$ transfer function $G_{p}(s)$ of order $n$. Let $C(s)$ be any dynamic stabilizing output feedback controller. Then

$$
G_{a}(s)=G_{p}(s)+C^{-1}(s)
$$

is ASPR if $C^{-1}(s)$ is proper or strictly proper.

We can adapt the proof of (Bar-Kana, 1989; BarKana, 1986) to the fractional case.

\section{Main result}

At this stage we propose a fractional order feedforward configuration of the form

$$
F(s)=\frac{F_{p}}{\left(1+s / s_{0}\right)^{\alpha}},
$$

with a real fractional power $0<\alpha<1$, to improve the robustness of the adaptive algorithm in the presence of perturbations, as such systems do not amplify much these random signals. This configuration could be considered as the inverse of an improper fractional $\mathrm{PD}^{\mu}$ controller, which was used in control systems with good proven performances (Podlubny, 1999b).

We can formulate the main result of this paper as the following theorem.

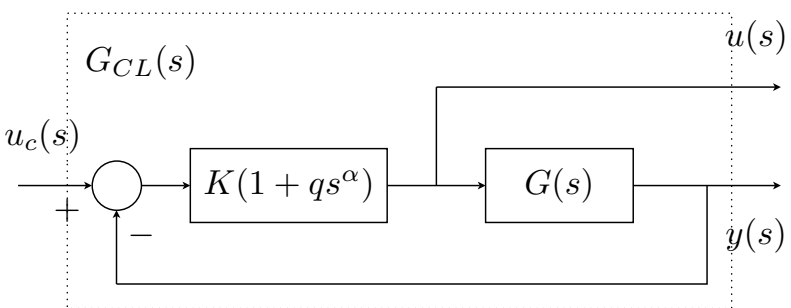

Fig. 1. Closed-loop system.

Theorem 1. Let $G(s)$ be any $m \times m$ strictly proper transfer matrix of an arbitrary MacMillan degree. $G(s)$ is not necessarily stable or minimum phase. Let

$$
H_{f}(s)=K\left(1+q s^{\alpha}\right)
$$

be some stabilizing controller for $G(s)$, represented in Fig. 1. Then the augmented controlled plant

$$
G_{a}^{f}(s)=G(s)+H_{f}^{-1}(s)=G(s)+\frac{K^{-1}}{1+q s^{\alpha}}
$$

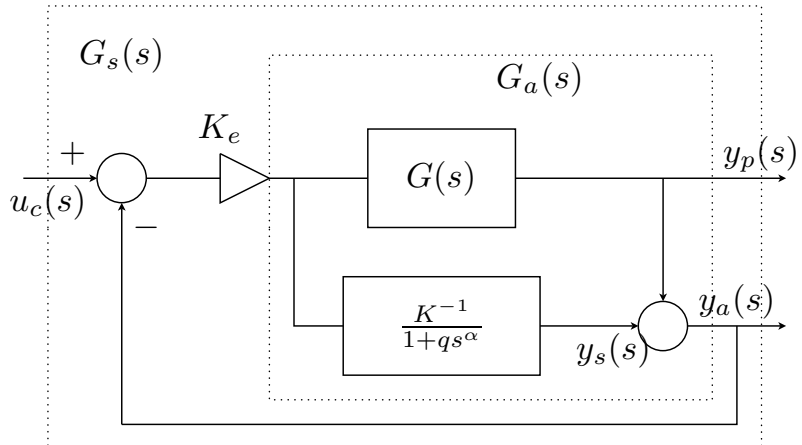

Fig. 2. Fictitious SPR configuration.

Proof. By Definition 6, if $G_{a}(s)$ is ASPR, then the closed-loop transfer function

$$
G_{c}(s)=\left[I+G_{a}(s) \tilde{K}_{e}\right]^{-1} G_{a}(s)
$$

is SPR for some positive definite constant gain matrix $\tilde{K}_{e}$.

Since $H_{f}^{-1}(s)$ from (14) is strictly proper (the relative degree $\alpha>0$ ), Lemma 2 implies that the augmented system $G_{a}^{f}(s)$ as defined in (15) is ASPR, which proves the theorem.

The stabilizing controller $H_{f}(s)$ can also be modelled as follows:

$$
H_{f}(s)=K(1+q s)^{\alpha} .
$$

From Definition 6 and the fact that the transfer function $G_{a}^{f}(s)$ is ASPR, we know that it can be stabilized by a gain $\tilde{K}_{e}$. Figure 2 illustrates the feedforward configuration. In addition, the stabilization is robust and holds for any gain $K_{e}>\tilde{K}_{e}$

Many previous works (Podlubny, 1999b) proposed $\mathrm{PD}^{\mu}$ improper controllers of the form (14):

$$
C(s)=K_{p}+K_{d} s^{\alpha},
$$

which can stabilize many realistic plants for sufficiently high values of $K$. in (13):

The feedforward of the equivalent effect is chosen as

$$
F(s)=C^{-1}(s)=\frac{F_{p}}{\left(1+s / s_{0}\right)^{\alpha}}
$$

with $s_{0} \gg \omega_{n}$, where $\omega_{n}$ is the nominal frequency of the controlled process and $F_{p}=K^{-1}$, such that the augmented plant becomes

$$
G_{a}(s)=G_{p}(s)+F(s) .
$$

$K$ should be very large, so that $F_{p}$ represents small coefficients, guaranteeing that $G_{a}(s)$ is ASPR. During control design we can take $G_{a}(s) \approx G_{p}(s)$ as a practical approximation. 


\section{Implementation in the MRAC scheme}

Model Reference Adaptive Control (MRAC) is one of the most popular approaches to adaptive control, in which the desired performance is specified by the choice of a reference model. The adjustment of parameters is achieved by means of the error between the output of the plant and the model reference output. Let us introduce the basic ideas of this approach, presented in Fig. 3.

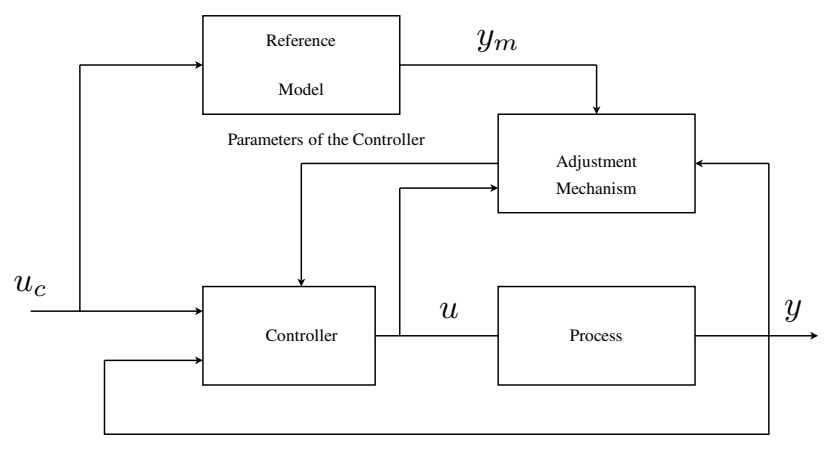

Fig. 3. Direct model reference adaptive control.

We consider a closed loop system where the controller has an adjustable parameter vector $\theta$. A model whose output is $y_{m}$ specifies the desired closed loop response. Let $e$ be the error between the closed loop system output $y$ and the model one $y_{m}$. One possibility is to adjust the parameters such that the cost function

$$
J(\theta)=\frac{1}{2} e^{2}
$$

is minimised. In order to make $J$ small, it is reasonable to change parameters in the direction of the negative gradient $J$, i.e.,

$$
\frac{\mathrm{d} \theta}{\mathrm{d} t}=-\gamma \frac{\partial J}{\partial \theta}=-\gamma e \frac{\partial e}{\partial \theta}
$$

or

$$
\frac{\mathrm{d} \theta}{\mathrm{d} t}=\gamma \varphi e,
$$

where $\varphi=-\partial e / \partial \theta$ is the regression (or measure) vector and $\gamma$ is the adaptation gain. This aproach is called the M.I.T. rule.

The introduction of simple feedforward in the MRAC adaptation loop as represented in Fig. 4 improves the robust stability performance against the controller gain fluctuations in the presence of perturbation and noise (Naceri and Abida, 2003). Previous works (Sobel and Kaufman, 1986) showed that the ASPR property of a process allows the implementation of very simple adaptive controllers that guarantee robust stability of the closed loop in the presence of bounded input or output disturbances.

The feedforward transfer function is chosen in much the same way as in (13), where the gain $F_{p}$ is a small coefficient.
Furthermore, to improve the robustness of this improved adaptive control scheme, we will use the fractional order parameter adaptation law proposed in (Ladaci and Charef, 2006) instead of (21), which is given by

$$
\frac{\mathrm{d}^{\beta} \theta}{\mathrm{d} t^{\beta}}=\gamma \varphi e
$$

where $\beta$ is a real number such that $0<\beta<2$.

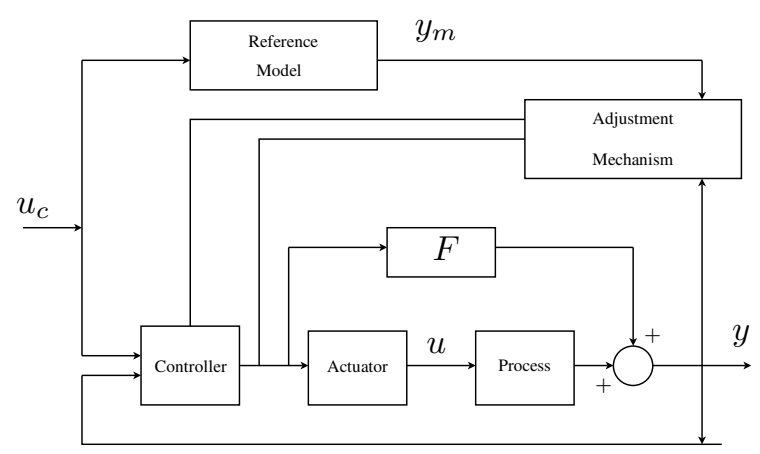

Fig. 4. Simple feedforward in the MRAC scheme.

\section{Simulation example}

Without any loss of generality we will apply this robust adaptive control method, both in the case of integer and fractional order feedforward, to an SISO model of a DC motor controlled for velocity, as given by (Zelmat, 2001),

$$
G_{p}(s)=\frac{2068.014706}{s^{2}+83.37811 s+2429.136291},
$$

and an actuator model of the form

$$
A(s)=\frac{1.05}{(1+s / 0.42)^{2}} .
$$

The plant is subject to random input and output disturbances of amplitudes 2 and 0.05 , respectively.

6.1. Integer order feedforward case. The classical MRAC structure is used here ( $\AA$ ström and Wittenmark, 1995). The reference model $G_{m}$ is given by

$$
G_{m}(s)=\frac{\omega_{n}^{2}}{s^{2}+2 \xi \omega_{n} s+\omega_{n}^{2}},
$$

where $\omega_{n}=11$ and $\xi=0.625$.

The feedforward transfer fuction $F$ is given by

$$
F(s)=\frac{0.001}{s+500}
$$

With the standard adaptation rule (21) and a regulation parameter $\gamma=0.001$, we obtain the results shown in Fig. 5. 
6.2. Fractional order feedforward case. In this case we use the fractional order MRAC structure (Ladaci and Charef, 2006) and the modified fractional order adaptive law (22) given by

$$
\frac{\mathrm{d}^{\beta} \theta}{\mathrm{d} t^{\beta}}=\gamma \varphi e,
$$

where $\beta=0.55$.

The fractional order reference model $G_{f}$ is given by

$$
G_{f}(s)=\frac{\left(\omega_{n}^{2}\right)^{\mu}}{\left(s^{2}+2 \xi \omega_{n} s+\omega_{n}^{2}\right)^{\mu}},
$$

where $\omega_{n}=11, \xi=0.625$ and $\mu=0.45$.

In our approach we have to use an integer order model approximation of the fractional order feedforward model in order to implement the adaptation algorithm. To this end, we have used the so-called singularity function method (Charef, Sun, Tsao and Onaral, 1992).

The fractional transfer function (29) is approximated with a tolerated approximation error of $2 \mathrm{~dB}$, to a rational transfer function $G_{f a}(s)$ given by (28).

The fractional order feedforward transfer function $F$ is chosen with the same gain and time constant of (26). The fractional order pole power is chosen arbitrarily here for illustration $(\alpha=0.6)$, giving a new design parameter. We get

$$
F(s)=\frac{0.001}{(s+500)^{0.6}}=\frac{0.001}{(500)^{0.6}} \frac{1}{(1+s / 500)^{0.6}} .
$$

It is approximated using the singularity function method with a tolerated approximation error of $2 \mathrm{~dB}$, to a linear second order transfer function $F_{a}(s)$ given by

$$
F(s) \approx F_{a}(s)=\frac{0.001}{(500)^{0.6}} \frac{1+s / z_{0}}{\left(1+s / p_{0}\right)\left(1+s / p_{1}\right)}
$$

with

$$
\begin{aligned}
& z_{0}=2320.79, \\
& p_{0}=733.90, \\
& p_{1}=5000 .
\end{aligned}
$$

With a regulation parameter $\gamma=10^{-8}$, we obtain the results of Fig. 6 .

\subsection{Remarks.}

- The command signal $u$ is smoother in the fractional case, which is a very useful property in the regulation problem.

- The proposed fractional order configuration of feedforward maintains stability and improves the level of performances, which confirms the advantage of the integrating fractional strategy in robust adaptive control.

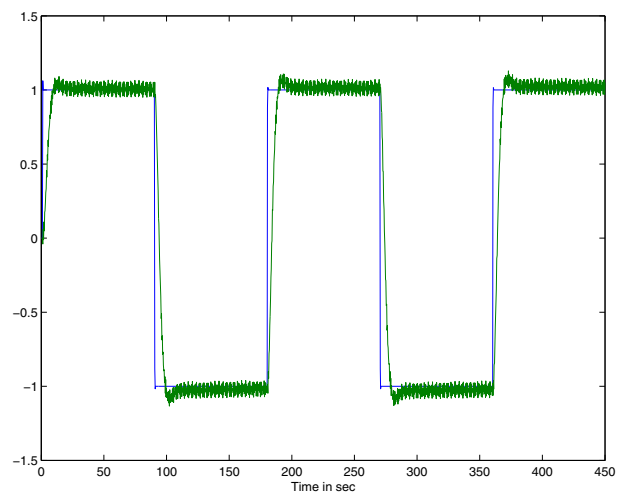

(a)

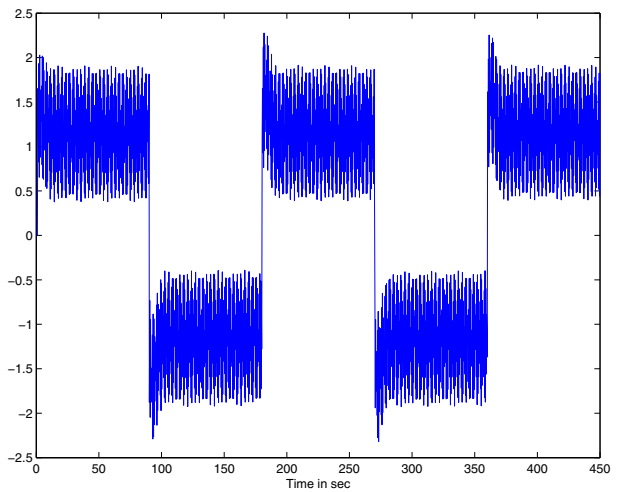

(b)

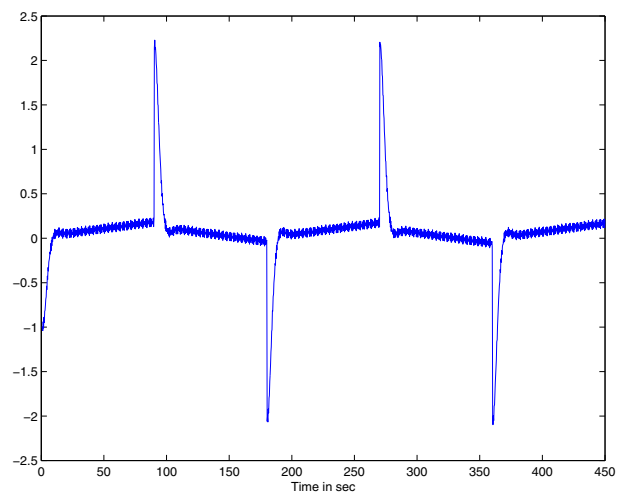

(c)

Fig. 5. Process output with integer feedforward: (a) process output $y(t)$, (b) control signal $u(t)$, (c) error signal $e(t)$.

- The regulation parameter is smaller in the case of the fractional order adaptive control scheme $(\gamma=$ $\left.10^{-8}\right)$ than in the integer order one $(\gamma=0.001)$, which improves the stability situation of the adaptive control system (Ladaci and Charef, 2006; Vinagre et al., 2002).

- The introduction of the fractional order feedforward transfer function gives new free design parameters $(\alpha$ in (13), $\beta$ in (22) and $\mu$ in (27) ) to improve the control system behaviour. 


$$
G_{f}(s) \approx G_{f a}(s)=\frac{0.0008264 s^{2}+0.1 s+1}{1.47210^{-9} s^{4}+4.53410^{-5} s^{3}+0.009071 s^{2}+0.1526 s+1} .
$$

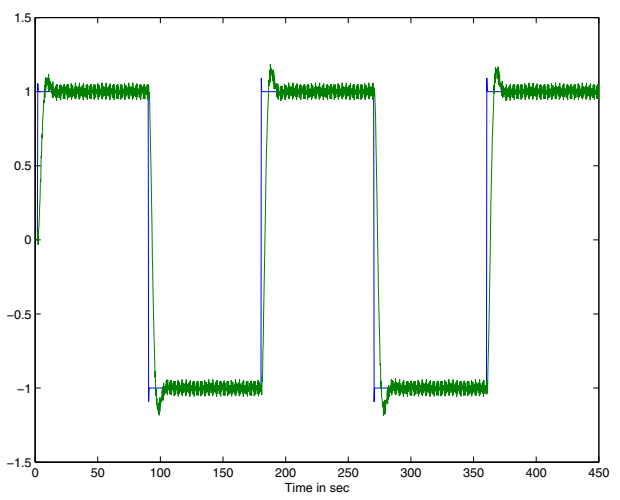

(a)

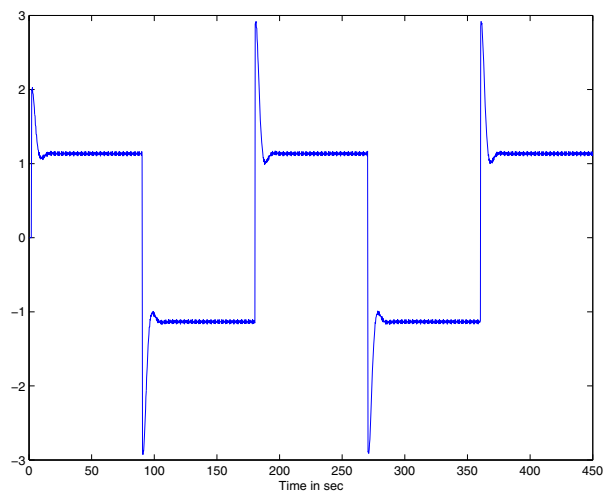

(b)

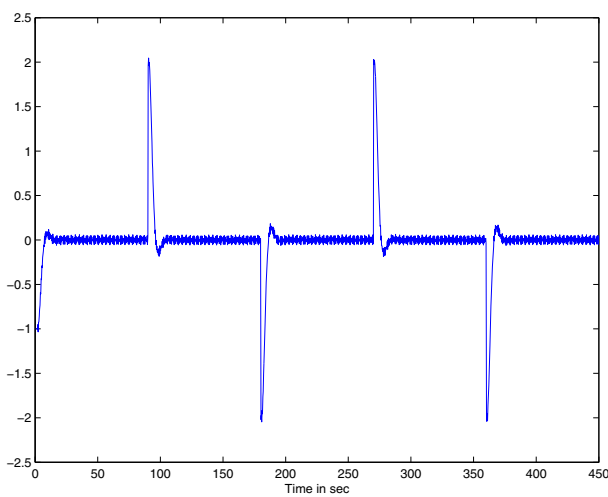

(c)

Fig. 6. Process output with fractional feedforward: (a) process output $y(t)$, (b) control signal $u(t)$, (c) error signal $e(t)$.

\section{Conclusion}

In this paper we have presented a new robust adaptive control strategy, by introducing a simple fractional feedforward configuration in the MRAC algorithm. The concept of the positive realness condition which is the basis of this robust control strategy is extended to fractional order control systems. The idea was to get benefit from the high performance quality of fractional order systems confirmed in many precedent research works. The stability proof of this adaptive control scheme developed for integer order filters in the control literature still holds for such systems. Simulation results revealed a better filtering ability of command and output signals, and more robustness against additive disturbances and noise than in the integer order feedforward configuration case.

\section{References}

Anderson, B. D. and Vongpanitlerd, S. (1973). Network Analysis and Synthesis, Prentice-Hall, Englewood Cliffs, NJ.

Åström, K. J. and Wittenmark, B. (1995). Adaptive Control, Addison-Wesley, Reading, MA.

Bar-Kana, I. (1986). Positive realness in discrete-time adaptive control systems, International Journal of Systems Science 17(7): 1001-1006.

Bar-Kana, I. (1987). Parallel feedforward and simplified adaptive control, International Journal Adaptive Control and Signal Processing 1(2): 95-109.

Bar-Kana, I. (1989). On positive realness in multivariable stationary linear systems, Proceedings of the Conference on Information Sciences and Systems, Baltimore, MD, USA.

Bar-Kana, I. and Kaufman, H. (1985). Global stability and performance of a simplified adaptive algorithm, International Journal of Control 42(6): 1491-1505.

Brin, I. A. (1962). On the stability of certain systems with distributed and lumped parameters, Automation and Remote Control 23: 798-807.

Charef, A. (2006). Analogue realisation of fractional-order integrator, differentiator and fractional $P I^{\lambda} D^{\mu}$ controller, IEE Proceedings-Control Theory and Applications 153(6): 714-720.

Charef, A., Sun, H. H., Tsao, Y. Y. and Onaral, B. (1992). Fractal system as represented by singularity function, IEEE Transactions on Automatic Control 37(9): 1465-1470.

Desoer, C. A. and Vidyasagar, M. (1975). Feedback Systems: Input-Output Properties, Academic Press, New York, NY.

Ioannou, P. and Sun, J. (1996). Robust Adaptive Control, Prentice Hall, Englewood Cliffs, NJ.

Kwan, C., Dawson, D. M. and Lewis, F. L. (2001). Robust adaptive control of robots using neural network: Global stability, Asian Journal of Control 3(2): 111-121.

Ladaci, S. and Charef, A. (2006). On fractional adaptive control, Nonlinear Dynamics 43(4): 365-378.

Ladaci, S., Loiseau, J. J. and Charef, A. (2008). Fractional order adaptive high-gain controllers for a class of linear systems, Communications in Nonlinear Science and Numerical Simulations 13(4): 707-714. 
Ladaci, S. and Moulay, E. (2008). $L^{p}$-stability analysis of a class of nonlinear fractional differential equations, International Journal of Automation and Systems Engineering 2(1): 4047.

Landau, Y. D. (1979). Adaptive Control: The Model Reference Approach, Marcel Dekker, New York, NY.

Miller, K. S. and Ross, B. (1993). An Introduction to the Fractional Calculus and Fractional Differential Equations, Wiley Interscience, New York, NY.

Naceri, F. and Abida, L. (2003). A novel robust adaptive control algorithm for AC drives, Computers and Electrical Engineering 29: 523-534.

Oustaloup, A. (1991). La commande CRONE, Hermès, Paris, (in French).

Oustaloup, A., Sabatier, J. and Moreau, X. (1998). From fractal robustness to the crone approach, ESAIM: Proceedings, Fractional Differential Systems: Models, Methods and Applications 5: 177-192.

Podlubny, I. (1999a). Fractional Differential Equations, Academic Press, New York, NY.

Podlubny, I. (1999b). Fractional order systems and $\mathrm{PI}^{\lambda} \mathrm{D}^{\mu}$ controllers, IEEE Transactions on Automatic Control 44(1): 208-214.

Sabatier, J., Oustaloup, A., Iturricha, A. and Lanusse, P. (2002). Crone control: Principles and extension to time-variant plants with asymptotically constant coefficients, Nonlinear Dynamics 29: 363-385.

Shaked, U. (1977). The zero properties of linear passive systems, IEEE Transactions on Automatic Control 22(6): 973-976.

Sobel, K. and Kaufman, H. (1986). Direct model reference adaptive control for a class of MIMO systems, Control and Dynamic Systems 24: 973-976.

Sun, H. and Charef, A. (1990). Fractal system-A time domain approach, Annals of Biomedical Engineering 18: 597-621.

Vinagre, B., Petras,I. and Chen, Y. (2002). Using fractional order adjustment rules and fractional order reference models in model-reference adaptive control, Nonlinear Dynamics 29: $269-279$.

Zelmat, M. (2001). Commande Modale et Adaptative, OPU, Algiers.

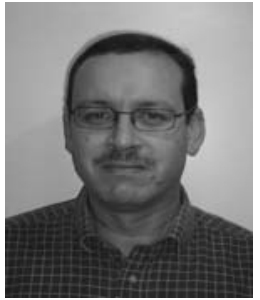

Samir Ladaci was born in Constantine, Algeria, in 1971. He obtained his engineer degree in automatic control in 1995 from the Polytechnic School of Algiers and his M.Sc. degree in industrial automation from the University of Annaba, Algeria, in 1999. He obtained his Ph.D. degree from the Department of Electronics, University of Constantine, Algeria, in 2007. He joined the University of Skikda in 2001 as an assistant professor. He paid numerous short visits to IRCCyN (Nantes, France) as an invited researcher from 2002 to 2008. His research interests include fractional order systems, fractional order control and adaptive control.

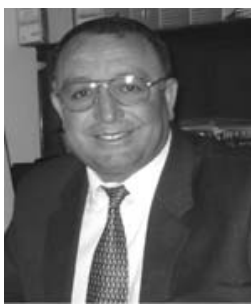

Abdelfatah Charef was born in OuedAthménia, Algeria, in 1961. He received the Diploma of Higher Education in electronics from the University of Constantine, Algeria, in 1984, the M.S. and Ph.D degrees in electrical engineering from Drexel University, Philadelphia, Pennsylvania, USA, in 1987 and 1991, respectively. In 1991, he joined the Department of Electronics of the University of Constantine, Algeria, where he is currently a professor and the director of the Signal Processing Laboratory. His current research interests are in the areas of systems analysis, fractional order systems, control systems, fractional order control, and fractional calculus in biomedical signal processing.

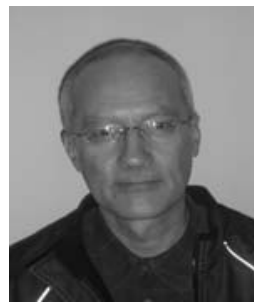

Jean Jacques Loiseau was born in Lorient, France, in 1958. He obtained the engineer degree in mechanics and the Ph.D. degree in automatic control in 1983 and 1986, respectively. From 1985 to 1990 he worked on ship building. He is presently a senior researcher of Centre National de la Recherche Scientifique at Institut de Recherche en Cybernétique de Nantes, where he has been since 1991. His research interests include the control and analysis of linear systems, time-delay systems, and discrete-event systems. He is the author of 33 journal papers, 12 book chapters or edited volumes, $88 \mathrm{com}$ munications at conferences. He is a co-editor-in-chief of Journal Européen des Systémes Automatisés (JESA) as well as a member of the IFAC Technical Committee TC2.2, Linear Control Systems, and TC1.3, Discrete Event and Hybrid Systems.

Received: 27 February 2008

Revised: 23 May 2008 